\title{
Parametric estimation of risk neutral density functions
}

\author{
Maria Grith* \\ Volker Krätschmer**
}

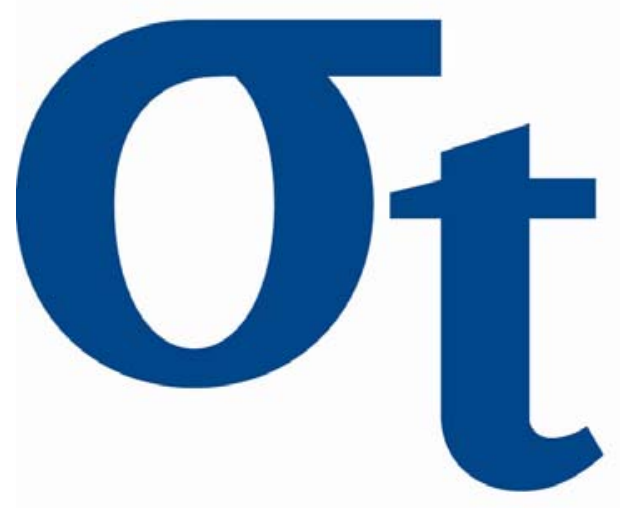

This research was supported by the Deutsche Forschungsgemeinschaft through the SFB 649 "Economic Risk". 


\title{
Parametric estimation of risk neutral density functions*
}

\author{
Maria Grith ${ }^{1}$ and Volker Krätschmer ${ }^{2}$ \\ 1 Research Associate at Humboldt-Universität zu Berlin, Ladislaus von \\ Bortkiewicz Chair of Statistics, Spandauer Straße 1, 10178 Berlin, Germany, \\ Email: gritmari@wiwi.hu-berlin.de \\ 2 Weierstrass Institute of Applied Analysis and Stochastics, Mohrenstrasse 39, \\ D-10117 Berlin, Germany; e-mail: kraetsch@wias-berlin.de.
}

Summary. This chapter deals with the estimation of risk neutral distributions for pricing index options resulting from the hypothesis of the risk neutral valuation principle. After justifying this hypothesis, we shall focus on parametric estimation methods for the risk neutral density functions determining the risk neutral distributions. We we shall differentiate between the direct and the indirect way. Following the direct way, parameter vectors are estimated which characterize the distributions from selected statistical families to model the risk neutral distributions. The idea of the indirect approach is to calibrate characteristic parameter vectors for stochastic models of the asset price processes, and then to extract the risk neutral density function via Fourier methods. For every of the reviewed methods the calculation of option prices under hypothetically true risk neutral distributions is a building block. We shall give explicit formula for call and put prices w.r.t. reviewed parametric statistical families used for direct estimation. Additionally, we shall introduce the Fast Fourier Transform method of call option pricing developed in [6]. It is intended to compare the reviewed estimation methods empirically.

Keywords: Risk neutral valuation principle, risk neutral distribution, logprice risk neutral distribution, risk neutral density function, Black Scholes formula, Fast Fourier Transform method, log-normal distributions, mixtures of log-normal distributions, generalized gamma distributions, model calibration, Merton's jump diffusion model, Heston's volatility model.

JEL classification: C13, C16, G12, G13.

* We are grateful to the Deutsche Forschungsgemeinschaft which has supported
this research via SFB 649 Ökonomisches Risiko, Humboldt-Universität zu Berlin. 


\section{Introduction}

It is usual practice of empirical studies on index option pricing in financial markets to start with the hypothesis of risk neutral valuation principle. That means it is assumed that prices of path independent derivatives with expiration at maturity may be represented as expected pay offs. The underlying distribution is referred as the risk neutral distribution. In the seminal paper [5], a stochastic model for financial markets has been established where this risk neutral distribution may be singled out by arbitrage arguments up to the volatility parameter. This Black Scholes model is nowadays very well understood, and widely used in financial industries due to the derived formula of risk neutral index call and put option prices.

Several empirical studies had come to the conclusion that the stochastic assumptions underlying the Black Scholes model does not fit very well the observed dynamics of asset prices. Therefore several alternative stochastic models have been proposed in the literature where typically risk neutral distributions may not be obtained by arbitrage arguments alone. However, within quite general stochastic frameworks one may identify theoretically risk neutral distributions compatible with observable liquid derivatives like call and put options. These risk neutral distributions are often called implied risk neutral distributions.

Compared to the risk neutral distribution according to the Black Scholes model implied risk neutral distributions generally do not have further specifications in advance. This complicates estimations in two directions. From the point of view of accuracy specification aspects like the choice of statistical families for the risk neutral distributions or the assumptions on stochastic models for the asset price processes have to be taken into account when selecting the estimation method and controlling the accuracy. Additionally the numerical problems associated with the implementation of the estimation method typically became more involved.

As a general assumption within the literature on estimation of risk neutral distributions they are considered as continuous distributions. The object is then to estimate related probability density functions called the risk neutral density functions, with a slight abuse of mathematical correctness. Two principal ways to estimate risk neutral density functions may be pointed out, parametric and nonparametric methods. This chapter deals with the parametric ones. One class of them is built upon parametric statistical families assumed to describe the risk neutral distribution accurately. The problem reduces to the estimation of the distribution parameters. The other group of methods estimate the probability density functions indirectly. A parametric stochastic model is assumed for the asset price processes, and the risk neutral density functions are extracted then after the calibration of the model to observed option prices. The chapter is organized as follows.

We shall start with the risk neutral valuation principle. There are controversial standpoints concerning the reasonability of this principle. Since the 
field of mathematical finance is mainly built upon the framework of arbitrage theory, many mathematicians accept risk neutral pricing for replicable options only. Instead non-linear pricing rules like superhedging are favoured which reduce to risk neutral pricing for replicable options. In section 2 we shall present an argumentation which might reconcile the different views.

The general idea behind the estimation methods under considerations is to fit option prices calculated under hypothetically true risk neutral density distributions to respective observed ones. Therefore these calculations play an important role for the implementation of the estimation methods. In section 4 we shall assume particular statistical families to model the risk neutral distributions. The considered families, namely log-normal distributions, mixtures of log-normal distributions and general gamma distributions, allow for explicit formula of call and put prices. Section 3 deals with calculations of call prices based on parametric stochastic models for the asset price processes. There the classical Black Scholes formula will be reviewed, and the Fast Fourier Transform method developed in [6] will be introduced. This method might be used as a tool for the model calibration as presented in section 5 . There it will also be shown how to extract the risk neutral density functions via Fourier methods. The whole line of reasoning will be explified by Merton's jump diffusion and Heston's volatility model. In the last section it is intended to compare the different reviewed estimation methods empirically.

\section{The risk neutral valuation principle}

Let $[0, T]$ be the time interval of investment in the financial market, where $t=0$ denotes the present time and $t=T \in] 0, \infty[$ the time of maturity.

Furthermore it is assumed that a riskless bond with constant interest rate $r>-1$ and a risky asset are traded in the financial market as basic underlyings. The evolution of the risky asset is expressed in terms of a state dependent nonnegative price process $\left(S_{t}\right)_{t \in[0, T]}$ with constant $S_{0}$. Notice that time discrete modelling may be subsumed under this framework.

For the pricing of nonnegative derivatives $\psi\left(S_{T}\right)$ it is often assumed that the risk valuation principle is valid. That means that there is a stochastic model for $\left(S_{t}\right)_{t \in[0, T]}$ by means of a probability measure Q such that the price of any $\psi\left(S_{T}\right)$ is characterized by

$$
\mathbb{E}_{\mathrm{Q}}\left[e^{-r T} \psi\left(S_{T}\right)\right] .
$$

There exist many arguments supporting this principle. From the viewpoint of the arbitrage theory it is closely related to the condition that Q is a so called martingale measure, i.e.

$$
\mathbb{E}_{\mathrm{Q}}\left[e^{-t r} S_{t} \mid S_{\tau}, \tau \leq \tilde{t}\right]=e^{-\tilde{t r}} S_{\tilde{t}} \text { for } 0 \leq \tilde{t}<t \leq T .
$$

In this case the financial market is arbitrage free in the sense that the value process $\left(V_{t}(H)\right)_{t \in[0, T]}$ of a self-financing investment strategy $H=\left(H_{t}\right)_{t \in[0, T]}$ 
which is bounded from below by $-\left(\delta S_{t}\right)_{t \in[0, T]}$ for some $\delta>0$, with $V_{0}(H) \leq 0$ the value at maturity $V_{T}(H)$ is vanishing almost surely if it is nonnegative. For a comprehensive account on the theory of arbitrage the reader is kindly referred to the monograph [10].

The expectation $\mathbb{E}_{\mathrm{Q}}\left[e^{-r T} \psi\left(S_{T}\right)\right]$ is then a so called arbitrage free price of $\psi\left(S_{T}\right)$, meaning that $\mathrm{Q}$ remains a martingale measure for the new financial market with an additional underlying having price process

$$
\left\{\mathbb{E}\left[e^{-r(T-t)} \psi\left(S_{T}\right) \mid S_{\tau}, \tau \leq t\right]\right\}_{t \in[0, T]} .
$$

Unfortunately, arbitrage free prices vary over the martingal measures unless a derivative $\psi\left(S_{T}\right)$ is replicable by the terminal wealth $V_{T}(H)$ of a value process $\left(V_{t}(H)\right)_{t \in[0, T]}$ of a self-financing investment strategy $H=\left(H_{t}\right)_{t \in[0, T]}$ satisfying boundness conditions as above. If every such derivative is replicable the financial market is called complete. An outstanding example is the famous Black-Scholes model (see below). However, at least in the special case of time discrete modelling complete financial markets are very exceptional, e.g. reducing directly to a binomial model within out setting (cf. [14], Theorem 5.38). Hence arbitrage arguments alone are not sufficient for a justification of the risk neutral valuation. Several suggestions have combined them with additional criteria. In [16] arbitrage free markets are embedded into a utility-based model for the terminal wealths of value processes of self-financing investment strategies that leads to risk neutral valuation of the derivatives $\psi\left(S_{T}\right)$. Another suggestion is built upon the observation that in organized markets call and put options are traded so often that they might be viewed as liquid derivatives. So the idea is to look for martingale measures Q consistent with observable prices $C(K)$ of call options with expiration $T$ and strike price $K$ in the sense

$$
C(K)=\mathbb{E}_{\mathrm{Q}}\left[e^{-r T} \max \left\{0, S_{T}-K\right\}\right] .
$$

If consistency is required for all strike prices $K$, then for any pair $\mathrm{Q}_{1}, \mathrm{Q}_{2}$ of such martingale measures the marginal distributions of $S_{T}$ w.r.t. $\mathrm{Q}_{1}, \mathrm{Q}_{2}$ coincide (see proof of Lemma 7.23 in [14]), implying $\mathbb{E}_{\mathrm{Q}_{1}}\left[e^{-r T} \psi\left(S_{T}\right)\right]=$ $\mathbb{E}_{\mathrm{Q}_{2}}\left[e^{-r T} \psi\left(S_{T}\right)\right]$ for a derivative $\psi\left(S_{T}\right)$. Moreover, there exist axiomatizations for pricing rules in financial markets that guarantee the existence of martingale measures which are consistent with the observable call prices $C(K)$ for all strikes $K$ (cf. e.g. [14], Proposition 7.26, [4]).

If the risk neutral valuation principle is valid w.r.t. to some stochastic model in terms of a probability measure $Q$, we shall call it risk neutral probability measure. As discussed above marginal distributions of $S_{T}$ are independent of the chosen risk neutral probability measure so that we may speak of the risk neutral distribution of $S_{T}$, henceforth denoted by $\mathrm{Q}_{S_{T}}$. Of course the marginal distributions of $\ln \left(S_{T}\right)$ are independent of the choice of risk neutral probability measures too, suggesting the convention of the logprice risk neutral distribution. We shall further restrict considerations to 
continuous risk neutral distributions admitting a probability density function $q$, which we shall call risk neutral density function. So from now on the assumption of the risk valuation principle should mean that the price of a derivative $\psi\left(S_{T}\right)$ is expressed by

$$
\int \psi(x) q(x) d x
$$

Since the risk neutral density function is unknown, the task is to estimate it upon observed prices for options $\psi\left(S_{T}\right)$ at time $t=0$. Typically, prices for call and put options are used. We shall review some widely used parametric methods. There always computations of hypothetical prices for options w.r.t. candidates of the risk neutral density functions are involved. For some models like the Black Scholes model such hypothetical prices for call and put options are given in implementable analytical expressions, for others like several stochastic volatility models numerically efficient ways of calculations have been developed. These results and methods will be the subject of the next section.

\section{Calculations of risk neutral option prices}

Let us assume that the stock price process $\left(S_{t}\right)_{t \in[0, T]}$ is characterized by a parameter vector $\vartheta \in \Theta \subseteq \mathbb{R}^{r}$ under the risk neutral probability measures. In the special case of the Black Scholes model the famous Black Scholes formulas provide explicit formulas for parameter dependent call and put prices. They will be reviewed in the following subsection. Afterwards we shall introduce the Fast Fourier Transform method to calculate call option prices as proposed in [6]. It relies on the additional assumption that the characteristic function of the log-price risk neutral distribution is known analytically.

\subsection{The Black Scholes formula}

In the Black Scholes model the price process $\left(S_{t}\right)_{t \in[0, T]}$ is modelled under the risk neutral probability measure by

$$
S_{t}=S_{0} \exp \left\{\left(r-\frac{\sigma^{2}}{2}\right) t+\sigma W_{t}\right\}
$$

where $\sigma>0$, and $\left(W_{t}\right)_{t \in[0, \infty[}$ denotes a standard Brownian motion. In particular $\vartheta \stackrel{\text { def }}{=} \sigma \in \Theta \stackrel{\text { def }}{=}] 0, \infty[$, the so called volatility, and the risk neutral distribution is a log-normal distributions with parameters $\mu \stackrel{\text { def }}{=}\left(r-\frac{\sigma^{2}}{2}\right) T+\ln \left(S_{0}\right)$ and $\sigma^{2} T$.

As usual, let $\Phi$ denote the distribution function of the standard normal distribution, and let $M_{K} \stackrel{\text { def }}{=} \frac{S_{T}}{K}$ be the moneyness w.r.t. strike price $K>0$. 
With these notations we may report the celebrated Black Scholes formula (cf. [5]) for the prices $C^{B S}(K, \sigma), P^{B S}(K, \sigma)$ of respectively the call and put with expiration at $T$ and strike price $K>0$ dependent on the volatility $\sigma$ :

$$
\begin{aligned}
& C^{B S}(K, \sigma)=S_{T} \Phi\left(d_{1}\right)-K e^{-r T} \Phi\left(d_{2}\right) \\
& P^{B S}(K, \sigma)=C_{B S}(K, \sigma)-S_{0}+e^{-r T} K \\
& d_{1} \stackrel{\text { def }}{=} \frac{-\ln (M)+T\left(r+\frac{\sigma^{2}}{2}\right)}{\sigma \sqrt{T}}, \quad d_{2} \stackrel{\text { def }}{=} d_{1}-\sigma \sqrt{T}
\end{aligned}
$$

\subsection{Fast Fourier Transform method to calculate call option prices}

We shall follow the line of reasoning in [6], assuming that the characteristic function $\Phi_{T \mid \vartheta}$ of the log-price risk neutral distribution is known analytically. Prominent examples are some widely used stochastic volatility models with or without jumps (see below). The aim is to calculate the hypothetical price $C^{\vartheta}(K)$ for the call option with expiration at $T$ and strike $K$ if $\vartheta$ is the true parameter vector driving the risk neutral model for the stock price process.

Recall that for an integrable function $f: \mathbb{R} \rightarrow \mathbb{R}$ we may define the so called Fourier transform $\hat{f}$ of $f$ via

$$
\hat{f}(y) \stackrel{\text { def }}{=} \int f e^{i y v} d v .
$$

Due to Plancherel's theorem (cf. [23], Theorem 9.13) we may recover $f$ from its fourier transform by

$$
f(x)=\int \frac{e^{-i x y} \hat{f}(y)}{2 \pi} d y
$$

if $f$ is in addition square integrable. Under the assumption

$$
\mathbb{E}\left[S_{T}^{1+\alpha}\right]<\infty \text { for some } \alpha>0
$$

this relationship between functions and their fourier transforms may be applied to

$$
C_{\alpha}^{\vartheta}: \mathbb{R} \rightarrow \mathbb{R}, x \mapsto e^{\alpha x} C^{\vartheta}\left(e^{x}\right)(\vartheta \in \Theta)
$$

(see [6]). We obtain the following formulas for the fourier transforms $\widehat{C_{\alpha}^{\vartheta}}$ of $C_{\alpha}^{\vartheta}(\vartheta \in \Theta)$

$$
\widehat{C_{\alpha}^{\vartheta}}=\frac{e^{-r T} \Phi_{T \mid \vartheta}(y-(1+\alpha) i)}{\alpha^{2}+\alpha-y^{2}+i(2 \alpha+1) y}
$$

A derivation may be found in [6] or [7], p. 189. This yields

$$
\begin{aligned}
C^{\vartheta}(K)=K^{-\alpha} C_{\alpha}^{\vartheta}(\ln (K)) & =\int^{\infty} \frac{K^{-\alpha} e^{-i y \ln (K)} \widehat{C_{\alpha}^{\vartheta}}(y)}{2 \pi} d y \\
& =\int_{0}^{\infty} \frac{K^{-\alpha} e^{-i y \ln (K)} \widehat{C_{\alpha}^{\vartheta}}(y)}{\pi} d y .
\end{aligned}
$$


The last equation holds because $C^{\vartheta}(K)$ is real, which implies that fourier transform $\widehat{C_{\alpha}^{\vartheta}}$ is odd in its imaginary part and even in its real part. Using the Trapezoid rule for the integral on the right hand side of (7), we may approximate the prices $C^{\vartheta}(K)$ by

$$
C^{\vartheta}(K) \approx \frac{1}{K^{\alpha} \pi} \sum_{j=0}^{N-1} e^{-i \eta j} \widehat{C_{\alpha}^{\vartheta}}(\eta j) \eta,
$$

where $\eta>0$ is the distance between the points of the integration grid. Bounds for sampling and truncation errors of this approximation have been developed in [19].

Approximation (8) suggests to apply the Fast Fourier algorithm which is an efficient algorithm to compute sums of the form

$$
w_{u}=\sum_{j=0}^{N-1} e^{-i \frac{2 \pi}{N} j u} z_{j} \text { for } u=0, \ldots, N-1
$$

(cf. [27]). In general, the strikes near the spot price $S_{0}$ are of interest because call options with such prices are traded most frequently. We thus consider an equidistant spacing of the $\log$-strikes around the $\log$ spot price $\ln \left(S_{0}\right)$ :

$$
x_{u}=-\frac{1}{N} N \zeta+\zeta u+\ln \left(S_{0}\right) \text { for } u=0, \ldots, N-1,
$$

where $\zeta>0$ denotes the distance between the log-strikes. Inserting (9) into formula (8) yields

$$
C^{\vartheta}\left\{\exp \left(x_{u}\right)\right\} \approx \frac{\exp \left(-\alpha x_{u}\right)}{\pi} \sum_{j=0}^{N-1} e^{-i \zeta \eta j u} e^{i \eta j\left\{\frac{1}{2} N \zeta-\ln \left(S_{0}\right)\right\}} \widehat{C_{\alpha}^{\vartheta}}(\eta j) \eta
$$

for $u=0, \ldots, N-1$. Now we may apply the Fast Fourier algorithm to

$$
z_{j} \stackrel{\text { def }}{=} e^{i \eta j\left\{\frac{1}{2} N \zeta-\ln \left(S_{0}\right)\right\}} \widehat{C_{\alpha}^{\vartheta}}(\eta j) \eta \text { for } j=0, \ldots, N-1
$$

provided $\zeta \eta=\frac{2 \pi}{N}$ holds. This restriction means on one hand that if we choose $\eta$ small in order to obtain a fine grid for the integration, we have a relatively large spacing between the log-strikes with few log-strikes lying around the desired region near $\ln \left(S_{0}\right)$. On the other hand a small $\zeta$ to catch many log-strikes near $\ln \left(S_{0}\right)$ a more rough grid for the integration is forced by the restriction. So we face a trade-off between accuracy and the number of interesting strikes. Accuracy may be improved for large $\eta$ by using better numerical integration rules. Carr and Madan considered the Simpson rule leading to the approximation

$$
\frac{\exp \left(-\alpha x_{u}\right)}{\pi} \sum_{j=0}^{N-1} e^{-i \zeta \eta j u} e^{i \eta j\left\{\frac{1}{2} N \zeta-\ln \left(S_{0}\right)\right\}} \widehat{C_{\alpha}^{\vartheta}}(\eta j) \frac{\eta}{3}\left\{3+(-1)^{j}-\delta_{0}(j)\right\}
$$


for $u=0, \ldots, N-1$, instead of $(10)$, where $\delta_{0}(0) \stackrel{\text { def }}{=} 1$ and $\delta_{0}(j) \stackrel{\text { def }}{=} 0$ for $j \neq 0$. The Fast Fourier algorithm may be applied to calculate

$$
z_{j} \stackrel{\text { def }}{=} e^{i \eta j\left\{\frac{1}{2} N \zeta-\ln \left(S_{0}\right)\right\}} \widehat{C_{\alpha}^{\vartheta}}(\eta j) \frac{\eta}{3}\left\{3+(-1)^{j}-\delta_{0}(j)\right\} \text { for } j=0, \ldots, N-1,
$$

again taking into account the condition $\zeta \eta=\frac{2 \pi}{N}$.

\section{Direct parametric estimation of the risk neutral density function}

The parametric approach to estimate the risk neutral density function directly starts with the assumption that the risk neutral distribution of $S_{T}$ belongs to a parametric family $W_{\Theta}\left(\Theta \subseteq \mathbb{R}^{r}\right)$ of one-dimensional continuous distributions. For any parameter vector $\vartheta \in \Theta$ and every strike price $K$ we may calculate the hypothetical prices for the call $C(K, \vartheta)$, the put $P(K, \vartheta)$ both with expiration $T$, and the forward $F_{\eta}$ by

$$
\begin{aligned}
& C(K \mid \vartheta)=e^{-r T} \int_{K}^{\infty}(x-K) q(x \mid \eta) d x \\
& P(K \mid \vartheta)=e^{-r T} \int_{0}^{K}(K-x) q(x \mid \eta) d x \\
& F_{\vartheta}=e^{-r T} \int_{0}^{\infty} x q(x \mid \vartheta) d x
\end{aligned}
$$

Therein, $q(\cdot \mid \vartheta)$ denotes any probability density function of the distribution from $W_{\Theta}$ associated with $\vartheta$.

The estimation of the risk neutral density function reduces to the estimation of the distribution parameter vector $\vartheta$. The most common approach is based on $S_{0}$, observed prices $Y_{1}, \ldots, Y_{n}$ for calls with strikes $K_{1}, \ldots, K_{m}$, and $\tilde{Y}_{1}, \ldots, \tilde{Y}_{m}$ with strikes $\tilde{K}_{1}, \ldots, \tilde{K}_{n}$. Both, calls and puts with expiration $T$. The parameter vector $\vartheta$ is estimated by minimizing the sum of the squared differences between the observed call, put and forward price and the hypothetical ones. More precisely, the estimation involves the solution of the following minimization problem

$$
\begin{aligned}
\min \sum_{i=1}^{m}\left\{Y_{i}-C\left(K_{i} \mid \vartheta\right)\right\}^{2} & +\sum_{j=1}^{n}\left\{\tilde{Y}_{i}-P\left(\tilde{K}_{i} \mid \vartheta\right)\right\}^{2} \\
& +\left(e^{-r T} S_{0}-F_{\vartheta}\right)^{2} \text { s.t. } \vartheta \in \Theta .
\end{aligned}
$$

The crucial step to implement this parametric approach is to find a proper statistical family $W_{\Theta}$ as a model for the risk neutral distribution. Usually, 
either a very general class a distribution is selected or mixtures of log-normal distributions are utilized. As general classes we shall discuss the benchmark case of log-normal distributions and the generalized Gamma distributions. Let us start with assumption of log-normal distributions.

\subsection{Estimation using log-normal distributions}

Closely related to the Black Scholes model the log-normal distributions are sometimes used for the risk neutral distribution, indicated as a benchmark case (cf. e.g. [18]). Recall that a probability density function $f_{\mathrm{LN}(\mu, \sigma)}$ of a $\log$-normal distribution with parameters $\mu \in \mathbb{R}$ and $\sigma>0$ is given by

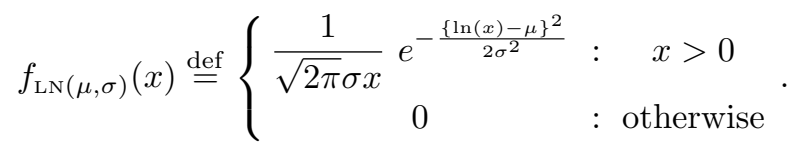

For fixed $\sigma^{2}$ and different $\mu_{1}, \mu_{2}$ the respective probability density functions $f_{\mathrm{LN}\left(\mu_{1}, \sigma\right)}$ and $f_{\mathrm{LN}\left(\mu_{2}, \sigma\right)}$ are linked by

$$
f_{\mathrm{LN}\left(\mu_{2}, \sigma\right)}=e^{\left(\mu_{1}-\mu_{2}\right)} f_{\mathrm{LN}\left(\mu_{1}, \sigma\right)}\left\{x e^{\left(\mu_{1}-\mu_{2}\right)}\right\} .
$$

Then applying the change of variables theorem for integration we obtain the following relationships between the call and put prices

$$
\begin{aligned}
& C\left(K \mid \mu_{2}, \sigma\right)=e^{\left(\mu_{2}-\mu_{1}\right)} C\left\{K e^{\left(\mu_{1}-\mu_{2}\right)} \mid \mu_{1}, \sigma\right\} \\
& P\left(K \mid \mu_{2}, \sigma\right)=e^{\left(\mu_{2}-\mu_{1}\right)} P\left\{K e^{\left(\mu_{1}-\mu_{2}\right)} \mid \mu_{1}, \sigma\right\} .
\end{aligned}
$$

The equations suggest to express prices $C(K \mid \mu, \sigma)$ and $P(K \mid \mu, \sigma)$ in terms of Black Scholes formulas, noticing that $C^{B S}(K, \sigma)=C\left\{K \mid\left(r-\frac{\sigma^{2}}{2}\right) T+\ln \left(S_{0}\right), \sigma\right\}$ and $P^{B S}(K, \sigma)=P\left\{K \mid\left(r-\frac{\sigma^{2}}{2}\right) T+\ln \left(S_{0}\right), \sigma\right\}$ holds for any strike $K$. For $\mu \in \mathbb{R}$ and $\sigma>0$ we obtain

$$
\begin{aligned}
& C^{B S}(K \mid \mu, \sigma) \stackrel{\text { def }}{=} C(K \mid \mu, \sigma) \\
& \left.=e^{\left\{\mu-\left(r-\frac{\sigma^{2}}{2}\right) T+\ln \left(S_{0}\right)\right.}\right\} C^{B S}\left\{K e^{\left(r-\frac{\sigma^{2}}{2}\right) T+\ln \left(S_{0}\right)-\mu}, \sigma\right\} \\
& P^{B S}(K \mid \mu, \sigma) \stackrel{\text { def }}{=} C(K \mid \mu, \sigma) \\
& \left.=e^{\left\{\mu-\left(r-\frac{\sigma^{2}}{2}\right) T+\ln \left(S_{0}\right)\right.}\right\} P^{B S}\left\{K e^{\left(r-\frac{\sigma^{2}}{2}\right) T+\ln \left(S_{0}\right)-\mu}, \sigma\right\} .
\end{aligned}
$$


With a slight abuse of convention we shall call $C^{B S}(K \mid \mu, \sigma)$ and $P^{B S}(K \mid \mu, \sigma)$ Black Scholes call and put prices too.

Next we want to introduce the approach to substitute log-normal distributions for the risk neutral distributions by mixtures of them.

\subsection{Estimation using log-normal mixtures}

The use of log-normal mixtures to model the risk neutral distribution of $S_{T}$ was initiated by [22] and became further popular even in financial industries by the studies [2], [20] and [26]. The idea is to model the risk neutral density function as a weighted sum of probability density functions of possibly different log-normal distribution. Namely, we set

$$
q\left(x \mid \mu_{1}, \ldots, \mu_{k}, \sigma_{1}, \ldots, \sigma_{k}, \lambda_{1}, \ldots, \lambda_{k}\right) \stackrel{\text { def }}{=} \sum_{i=1}^{k} \lambda_{i} f_{\mathrm{LN}\left(\mu_{i}, \sigma_{i}\right)}(x),
$$

where $f_{\mathrm{LN}\left(\mu_{i}, \sigma_{i}\right)}$ denotes a probability density function of the log-normal distribution with parameters $\mu_{i} \in \mathbb{R}$ as well as $\sigma_{i}>0$, and nonnegative weights $\lambda_{1}, \ldots, \lambda_{k}$ summing up to 1 .

This approach might be motivated w.r.t. two aspects. Firstly such density functions are flexible enough to model a great variety of potential shapes for the risk neutral density function. Secondly, we may compute easily the hypothetical call and put prices in terms of respective Black-Scholes formulas by

$$
\begin{aligned}
& C\left(K \mid \mu_{1}, \ldots, \mu_{k}, \sigma_{1}, \ldots, \sigma_{k}, \lambda_{1}, \ldots, \lambda_{k}\right)=\sum_{i=1}^{k} \lambda_{i} C^{B S}\left(K \mid \mu_{i}, \sigma_{i}\right) \\
& P\left(K \mid \mu_{1}, \ldots, \mu_{k}, \sigma_{1}, \ldots, \sigma_{k}, \lambda_{1}, \ldots, \lambda_{k}\right)=\sum_{i=1}^{k} \lambda_{i} P^{B S}\left(K \mid \mu_{i}, \sigma_{i}\right) .
\end{aligned}
$$

Additionally, drawing on well known formulas for the expectations of lognormal distributions, we obtain

$$
F_{\mu_{1}, \ldots, \mu_{k}, \sigma_{1}, \ldots, \sigma_{k}, \lambda_{1}, \ldots, \lambda_{k}}=\sum_{i=1}^{k} \lambda_{i} e^{\left(\mu_{i}+\frac{\sigma_{i}^{2}}{2}-r T\right)}
$$

Recalling that the parameter estimation is based on observations of $m$ call and $n$ put prices we have to take into account the problem of overfitting. More precisely, the number $3 k-1$ of parameters should not exceed $m+n$, the number of observations. Furthermore in order to reduce the numerical complexity of the minimization problem underlying the estimation it is often suggested to restrict estimation to the choice of $k \in\{2,3\}$.

Empirical evidence (cf. e.g. [9], [24] and [25]) shows that the implied skewness of the underlying used in options is often negative, in contrary to the 
skewness of log-normal distributions. In order to take into account negative skewness Savickas proposed to use Weibull distributions (cf. [24] and [25]). In [12] this suggestion has been extended to the family of generalized gamma distributions that will be considered in the next subsection.

\subsection{Estimation using generalized Gamma distributions}

According to $\vartheta \stackrel{\text { def }}{=}(\alpha, \beta, k) \in \Theta \stackrel{\text { def }}{=}] 0, \infty\left[{ }^{3}\right.$ a respective probability density function $f_{\mathrm{G}}(\cdot \mid \alpha, \beta, \delta)$ is given by

$$
f_{\mathrm{G}}(\cdot \mid \alpha, \beta, k)=\left\{\begin{array}{cc}
\frac{1}{\Gamma(k)}\left(\frac{\beta}{\alpha}\right)\left(\frac{x}{\alpha}\right)^{\beta k-1} \exp \left\{-\left(\frac{x}{\alpha}\right)^{\beta}\right\} & : \quad x>0 \\
0 & : \text { otherwise }
\end{array},\right.
$$

where $\Gamma$ denotes the Gamma function. The corresponding cumulative distribution function $G(\cdot \mid \alpha, \beta, k)$ is given by

$$
G(x \mid \alpha, \beta, k) \stackrel{\text { def }}{=} I\left\{k,\left(\frac{x}{\alpha}\right)^{\beta}\right\}
$$

where $I$ denotes the incomplete gamma function defined as

$$
I(k, y) \stackrel{\text { def }}{=} \frac{1}{\Gamma(k)} \int_{0}^{y} x^{k-1} e^{-x} d x .
$$

It is known that $k=1$ leads to a Weibull distribution, when $\beta=1$ we get a gamma distribution, when $\beta=k=1$ we obtain an exponential distribution and when $k \rightarrow \infty$ we arrive a log-normal distribution. Explicit formulas for the respective hypothetical prices $C(K \mid \alpha, \beta, k), P(K \mid \alpha, \beta, k)$ and $F_{\alpha, \beta, k}$, the moment generating function, have been derived in [12] (pp. 58,70). They read as follows.

$$
\begin{aligned}
& F_{\alpha, \beta, k}=\alpha \frac{\Gamma\left(k+\frac{1}{\beta}\right)}{\Gamma(k)} \\
& C(K \mid \alpha, \beta, k)=e^{-r T} F_{\alpha, \beta, k}-e^{-r T} K \\
& -\left[F_{\alpha, \beta, k} I\left\{k-\frac{1}{\beta},\left(\frac{K}{\alpha}\right)^{\beta}\right\}+K I\left\{k,\left(\frac{K}{\alpha}\right)^{\beta}\right\}\right] \\
& P(K \mid \alpha, \beta, k)=e^{-r T}\left[K I\left\{k,\left(\frac{K}{\alpha}\right)^{\beta}\right\}-F_{\alpha, \beta, k} I\left\{k+\frac{1}{\beta},\left(\frac{K}{\alpha}\right)^{\beta}\right\}\right] .
\end{aligned}
$$

A different class of methods to estimate the risk neutral density start with a parametric model of the whole stock price process which determines in an analytic way the risk neutral distribution. Then the risk neutral density will be estimated indirectly via calibration of the stock price process. 


\section{Estimation via calibration of the stock price process}

The starting point for the indirect estimation of the risk neutral density function via model calibration is the assumption that the risk neutral probability measure of the stock price process $\left(S_{t}\right)_{t \in[0, T]}$ is characterized by a parameter vector $\vartheta \in \Theta \subseteq \mathbb{R}^{r}$. Furthermore it is supposed that the characteristic functions $\Phi_{T \mid \vartheta}$ of $\ln \left(S_{T}\right)$ under $\vartheta$ is known analytically. Prominent examples are some widely used models (see below).

Based on observed prices $Y_{1}, \ldots, Y_{m}$ for call options with expiration $T$ and strike prices $K_{1}, \ldots, K_{m}$ the stock price process is calibrated to obtain an estimated parameter vector $\hat{\vartheta}$. A popular way is to solve the following inverse problem (cf. e.g. [3], [1])

$$
\begin{aligned}
& \min \sum_{i=1}^{m}\left\{Y_{i}-C^{\vartheta}\left(K_{i}\right)\right\}^{2} \\
& \text { s.t. } \vartheta \in \Theta,
\end{aligned}
$$

where $C^{\vartheta}\left(K_{i}\right)$ denotes the hypothetical call price with expiration $T$ and strike price $K_{i}$ if $\vartheta$ is the true characteristic parameter vector. These prices might be calculated via the Fast Fourier Transform method as introduced in subsection 3.2. This approach has the attractive numerical feature that for implementation we may draw on the Fast Fourier algorithm.

Once we have solved the inverse problem some parameter vector say $\hat{\vartheta}$, we might extract the risk neutral density function in the following way. Firstly we obtain by Fourier inversion theorem (cf. [11], 9.5.4) for probability density function $q_{\log \mid \hat{\vartheta}}$ of $\ln \left(S_{T}\right)$

$$
q_{\log \mid \hat{\vartheta}}(x)=\int \frac{\Phi_{T \mid \hat{\vartheta}}(y) e^{-i t y}}{2 \pi} d y .
$$

Then application of the transformation theorem for probability density functions yields the estimation

$$
q_{\hat{\vartheta}}(x)=\left\{\begin{array}{ccc}
\frac{q_{\log \mid \hat{\vartheta}(x)}}{x} & : & x>0 \\
0 & : & \text { otherwise }
\end{array} .\right.
$$

Let us now have a closer look at some special models where we shall identify the respective calibration parameter and characteristic functions. We shall consider refinements of the classical Black Scholes model. Namely Merton's jump diffusion model which incoporates possible large or sudden movement in prices, and Heston's volatility model which take into account state dependent changes in volatilities. 


\subsection{Merton's jump diffusion model}

The jumps of the log prices are usually modelled by a compound Poisson process $\sum_{i=1}^{N_{t}} Y_{i}$, consisting of a Poisson process $\left(N_{t}\right)_{t \in[0, \infty[}$ with intensity parameter $\lambda>0$ independent of a sequence $\left(Y_{i}\right)_{i \in \mathbb{N}}$ of i.i.d. random variables. The $N_{t}$ model the random number of jumps, whereas the respective jump sizes are expressed by the $Y_{i}$ having a common distribution of typical jump size. Within the Merton's jump diffusion model a normal distribution $N\left(\mu, \delta^{2}\right)$ is assumed as the distribution of typical jump size. Then this compound Poisson process is added to classical Black Scholes model. As introduced in [21], the risk neutral price process within Merton's jump diffusion model may be described by

$$
S_{t}=S_{0} \exp \left(\mu^{M} t+\sigma W_{t}+\sum_{i=1}^{N_{t}} Y_{i},\right)
$$

where $\mu^{M}=r-\frac{\sigma^{2}}{2}-\lambda\left\{\exp \left(\mu+\frac{\delta^{2}}{2}\right)-1\right\}, \sigma>0$, and $\left(W_{t}\right)_{t \in[0, \infty[}$ denoting a standard Brownian motion which is independent of the compound Poisson process.

Drawing on well-known formulas for characteristic functions of normally distributed random variables (cf. [11], Proposition 9.4.2), and that for compound Poisson processes ([8], Proposition 3.4), we obtain the characteristic function $\Phi_{\ln \left(S_{T}\right)}$ of $\ln \left(S_{T}\right)$ by an easy calculation, yielding

$$
\begin{aligned}
\Phi_{\ln \left(S_{T}\right)}(z) & =\exp \left\{i z \ln \left(S_{0}\right)\right\} \\
& \times \exp \left[T\left\{1-\frac{\sigma^{2} z^{2}}{2}+i \mu^{M} z+\lambda\left(e^{\left(-\frac{\delta^{2} z^{2}}{2}+i \mu z\right)}\right)\right\}\right]
\end{aligned}
$$

As parameter vector we may identify $\left.\vartheta \stackrel{\text { def }}{=}\left(\sigma^{2}, \lambda, \mu, \delta^{2}\right) \in\right] 0, \infty\left[{ }^{2} \times \mathbb{R} \times\right] 0, \infty[\stackrel{\text { def }}{=}$ $\Theta$.

\subsection{Heston's volatility model}

A popular approach to substitute the deterministic volatility in the Black Scholes model by a stochastic process $\left(v_{t}\right)_{t \in[0, \infty[}$. was proposed in [15]. In this model the risk neutral dynamics of the $\log$ price $\ln \left(S_{t}\right)$ is expressed by the stochastic differential equations

$$
\begin{aligned}
& d \ln \left(S_{t}\right)=\left(r-\frac{1}{2} v_{t}\right) d t+\sqrt{v_{t}} d W_{t}^{S} \\
& d v_{t}=\kappa\left(\eta-v_{t}\right) d t+\theta \sqrt{v_{t}} d W^{V},
\end{aligned}
$$

where $\left(W_{t}^{S}\right)_{t \in[0, \infty[},\left(W_{t}^{V}\right)_{t \in[0, \infty[}$ are correlated standard Brownian motion with rate $\rho$ : 


$$
\operatorname{Cov}\left(d W_{t}^{S}, d W_{t}^{V}\right)=\rho d t
$$

An analytical expression of the characteristic function $\Phi_{\ln \left(S_{T}\right)}$ of $\ln \left(S_{T}\right)$ has been derived in [15] in the following way

$$
\begin{aligned}
\Phi_{\ln \left(S_{T}\right)}(z) & =\frac{\exp \left[\frac{\kappa \eta T(\kappa-i \rho \theta z)}{\theta^{2}}+i z\left\{T r+\ln \left(S_{0}\right)\right\}\right]}{\left\{\cosh \left(\frac{\gamma T}{2}\right)+\frac{\kappa-i \rho \theta z}{\gamma} \sinh \left(\frac{\gamma T}{2}\right)\right\}^{\frac{2 \kappa \eta}{\theta^{2}}}} \\
& \times \exp \left\{-\frac{\left(z^{2}+i z\right) v_{0}}{\gamma \operatorname{coth}\left(\frac{\gamma T}{2}\right)+\kappa-i \rho \theta z}\right\},
\end{aligned}
$$

where $\gamma=\sqrt{\theta^{2}\left(z^{2}+i z\right)+(\kappa-i \rho \theta z)^{2}}$. As parameter vector we obtain

$$
\vartheta \stackrel{\text { def }}{=}(\theta, \rho, \kappa, \eta) \in] 0, \infty[\times[-1,1] \times[0, \infty[\times] 0, \infty[\stackrel{\text { def }}{=} \Theta .
$$

\section{Empirical study}

In this section we will demonstrate the methods exposed in the theoretical part and address some aspects of concern for practitioners. Estimating the risk neutral density by direct methods involves the choice of parametric distribution family to which it belongs to. This introduces some arbitrariness in modelling because the distribution family must be selected a priori from a set of candidates. Indirect modelling relies on assumptions about the data generating process and the shape of the risk neutral density is intrinsically related to the parameter values of the underlying process.

Practitioners are interested in modelling the RND from observed data and therefore have to solve an inverse problem. Model parameters are often obtained by solving nonlinear least squares equations for which analytical solutions may be very difficult or impossible to derive. Therefore, one has to rely on numerical optimization algorithms in order to retrieve the unknown parameters. In addition, the approaches may suffer the drawbacks associated with the ill-posedness of some inverse problems in pricing models: there may exist no solution at all or an infinity of solutions. The last case means that there are many sets of parameters reproducing call prices with equal precision, which in turn may translate in pricing errors with many local minima or flat regions with low model sensitivity to variations in parameters. The solutions are often very sensitive to the numerical starting values; numerical instability may also occur if the dependence of solutions to the observed data is discontinuous. Uniqueness and stability may be achieved by introducing a regularization method: e.g. adding penalty to the linear least squares term. For further discussions on regularization methods see [8].

In order to assess the shape of RND implied by different parametric approaches we use paired European call options written on the underlying DAX 
stock index which mature in one month (21 days) and strike prices observed on 20040121. The data is provided by Eurex - Deutsche Börse and collected from the Research Data Center (RDC) of the Collaborative Research Center 649. Strike prices have been transformed to account for intraday stock price movements; these have been computed from the futures prices following a methodology by [13]. The EURIBOR interpolated interest rate for this maturity is $r=2.05 \%$ per annum and the stock index value taken from the daily series DAX 30 Index is $S=4138$. The dividend rate is zero. Observations that do not respect general arbitrage conditions (see [17]) have been excluded from the sample. We are left with 2562 paired observations, which we display in figure 1. The counterpart representation of the observations in the implied volatility space (based on Black-Scholes valuation formula) will be further used to assess the quality of the estimates. Note that in practice, it is often more convenient to match implied volatilities which are of the same order of magnitude relative to call prices which display a much larger out-of-the-money variation.
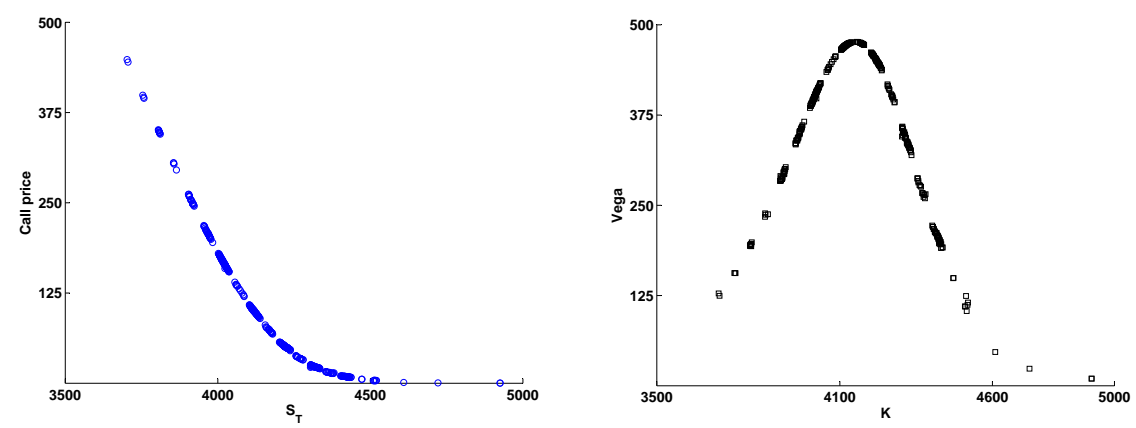

Fig. 1. Left: European call option versus strike prices on 20040121. Right: $\nu$ of the observed call prices.

Figure 2 depicts the estimation results for the RND by direct methods as well as the fit in the implied volatility space. In the upper left panel, the Black-Scholes log-normal RND depends on only one unknown for given risk free interest rate, the constant - across strikes - volatility parameter $\sigma$. It is contrasted with the implied volatility of the observed call prices in the right panel.

Next, we fit a mixture of log-normal densities. The parameter $k$ is usually assumed to be unknown and one has to apply appropriate criteria to find the optimal parameter value. Here, we illustrate the method for fixed $k=2$ in the central part of figure 2 . Since $\mu_{1}$ and $\mu_{2}$ are known up to the volatility parameters $\sigma_{1}$ and $\sigma_{2}$ respectively of the components, the mixing distribution will have three unknown parameters. We have investigated the shape of the 

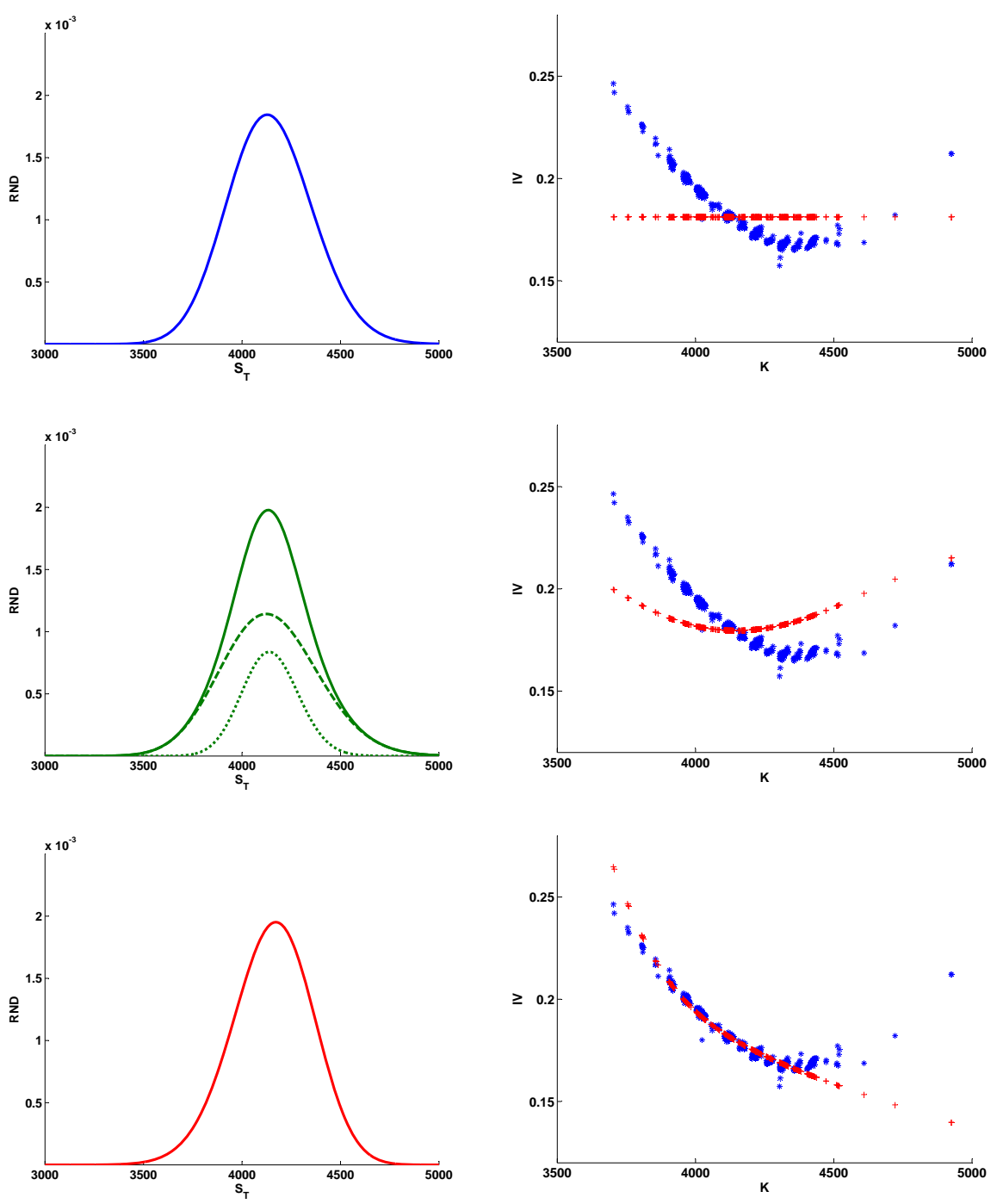

Fig. 2. Left: RND estimated by: log-normal distribution with $\sigma=0.18$ (top), mixture of log-normal distributions for weighted components $\sigma_{1}=0.24$ - dashed, $\sigma_{2}=0.15$ dotted with $\lambda=0.31$ (center) and generalized gamma distribution with $\alpha_{1}=3033.03$, $\beta_{2}=6.86$ and $k=9.05$ (bottom). Right: $I V_{B S}$ for observed call prices (asterisk) and fitted call prices (plus sign)

resulting density and found that it is robust with respect to the mixtures, in the sense that for known basic densities, the proportion parameter $\lambda$ regulates the relative impact of each component. Conversely, one can fix $\lambda$ and try to 
estimate $\sigma_{1}$ and $\sigma_{2}$. The mixture generates a rather symmetric smile, with a minimum different from that of the volatility skew of the observed prices. The higher kurtosis improves the fit at a price of higher skewness compared with the simple log-normal case. This shows that using mixtures of log-normals improves the fit especially by higher kurtosis. Every (central) moment of a linear combination of densities is given by the same combination of the corresponding moments. The third moment of a log-normal density is always positive, therefore a mixture of log-normal can never generate negative skewness. In order to generate a negative skew either other mixture types or other parametric models for the RND has to be considered.
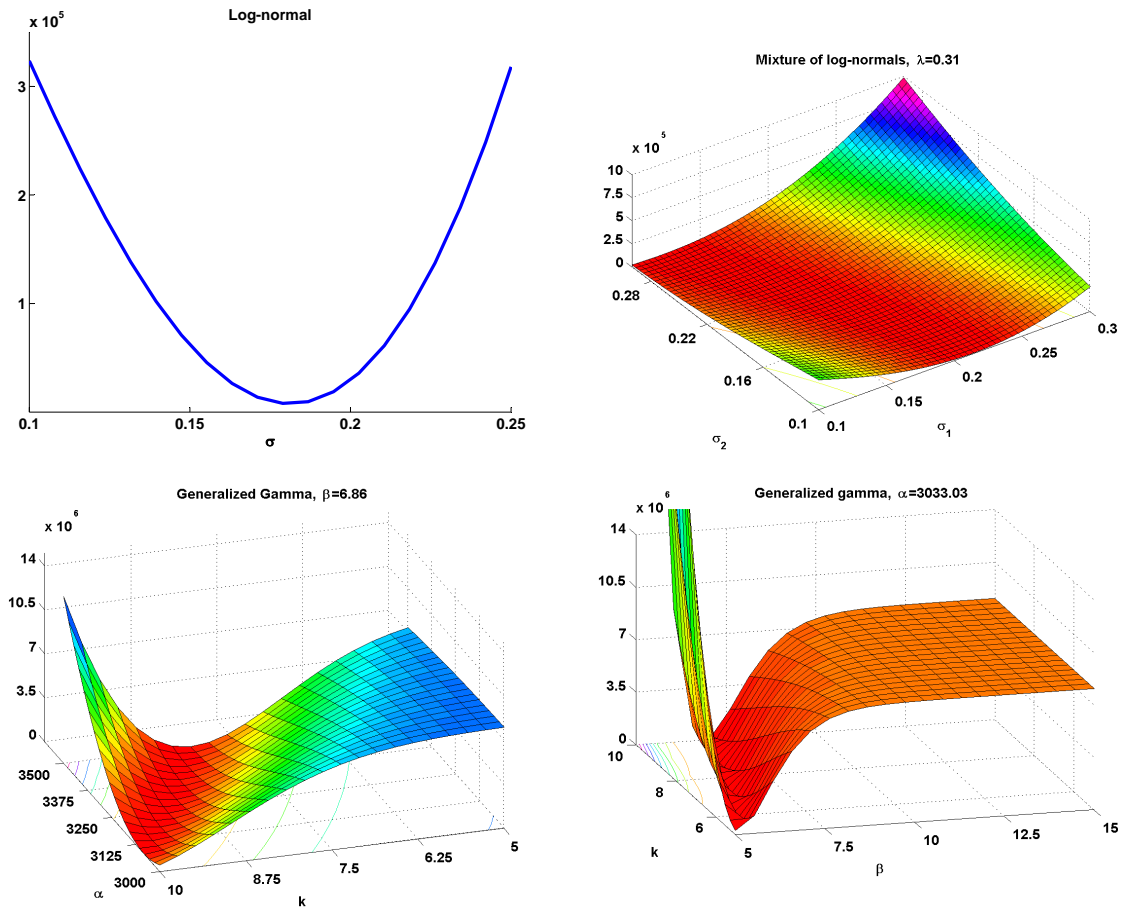

Fig. 3. Estimation error function by direct methods

Generalized gamma distribution allows more flexibility in modelling the shape of RND. It depends on three parameters: the parameter $\alpha$ is a scale parameter, $k$ is the index parameter and $\beta$ is the power parameter. There are many sets of parameters that give a good fit and produce relatively stable shapes of the RND. For a given set of parameters we display the results in the lower panel of figure 2 . In the implied volatility space, the gamma distribution cannot reproduce the smile; it establishes a negative relationship between the strike price and the implied volatility. In terms of the fitting errors this does 


\begin{tabular}{crrrrr}
\hline \hline Model & \multicolumn{4}{c}{ Mean St. Dev. Skewness Kurtosis } & RSS \\
\hline Log-Normal & 4145.11 & 216.99 & 0.15 & 3.04 & 7693.08 \\
Mixture & 4139.39 & 221.28 & 0.20 & 3.83 & 7465.22 \\
Generalized Gamma & 4152.76 & 205.44 & -0.18 & 3.06 & 351.07 \\
\hline \hline
\end{tabular}

Table 1. Comparison of the RND estimates by direct method in terms of moments and fit: log-normal for $\sigma=0.18$ (blue), mixture of lognormals for $\sigma_{1}=0.24, \sigma_{2}=0.15$ and $\lambda=0.31$, generalized gamma for $\alpha_{1}=3033.03, \beta_{2}=6.86$ and $k=9.05$

not constitute too much of a problem because the vega of the call price $\nu=\frac{\partial C}{\partial \sigma}$ decreases steeply for large $K$ and reaches values close to 0 for deep out-of-the money call prices (see figure 1 right). The vega of the call option based on the Black-Scholes's call pricing formula is given by $\nu=S \sqrt{T}\left(\phi\left(d_{1}\right)\right)$ with $d_{1}$ defined in equation 4.

For the Black-Scholes RND the calibration error function $\left\|Y-C^{\hat{\vartheta}}\right\|^{2}$, where $Y$ is the vector of observed and $C^{\hat{\vartheta}}$ the vector of fitted call prices (i.e. the objective function in (15)) has a unique minimum (see figure 3 upper panel left). The same holds for the mixture when the two basic densities are fixed. The RSS takes values close to a minimum for a multitude of combinations of $\sigma_{1}$ and $\sigma_{2}$ (see figure 3 right). The following two panels in figure 3 refer to the generalized gamma distribution. The objective function is a surface which forms a valley or rift of minimum values. This illustrate the ill-posed problem.

The pricing errors computed as a difference between observed and fitted call prices, display some regularities: RND-s estimated by the first two methods lead to underpriced calls for ITM options and overpriced calls for OTM options; the discrepancies diminish for deep ITM and OTM options. Generalized gamma distribution is flexible enough to give a good fit for a large range of strikes in the central part of the distribution. Since the observations in the tails are more sparse, the pricing errors will be higher for deep ITM call options. In this particular case, the estimated density will have fetter left tails resulting in overpriced options for small strike prices. However, the absolute pricing errors are smaller than for the other candidates. The resulting moments of the estimated densities are summarized in table 2 .

In the remaining of this section, we describe the results by the indirect approach for finding the RND. The calibration of the second type of models is further supported by advanced numerical methods available, such as Fast Fourier Transform (FFT). In order to apply the FFT-based algorithm we use the characteristic function of the risk neutral density as described in section 5 for the Merton and Heston models and set the parameters $\alpha=1.25, N=4096$, and $\eta=0.25$. For OTM option prices the calibration error increases; therefore, we use the Fourier Transform of OTM option prices as described in [6]. With the above parameters choice and pricing rules, we solve the problem of model calibration. This implies solving the minimization problem given in (26) and 
(27). We describe the results for both models in terms of the resulting RND and fit in the $I V$ space in figure 4 .
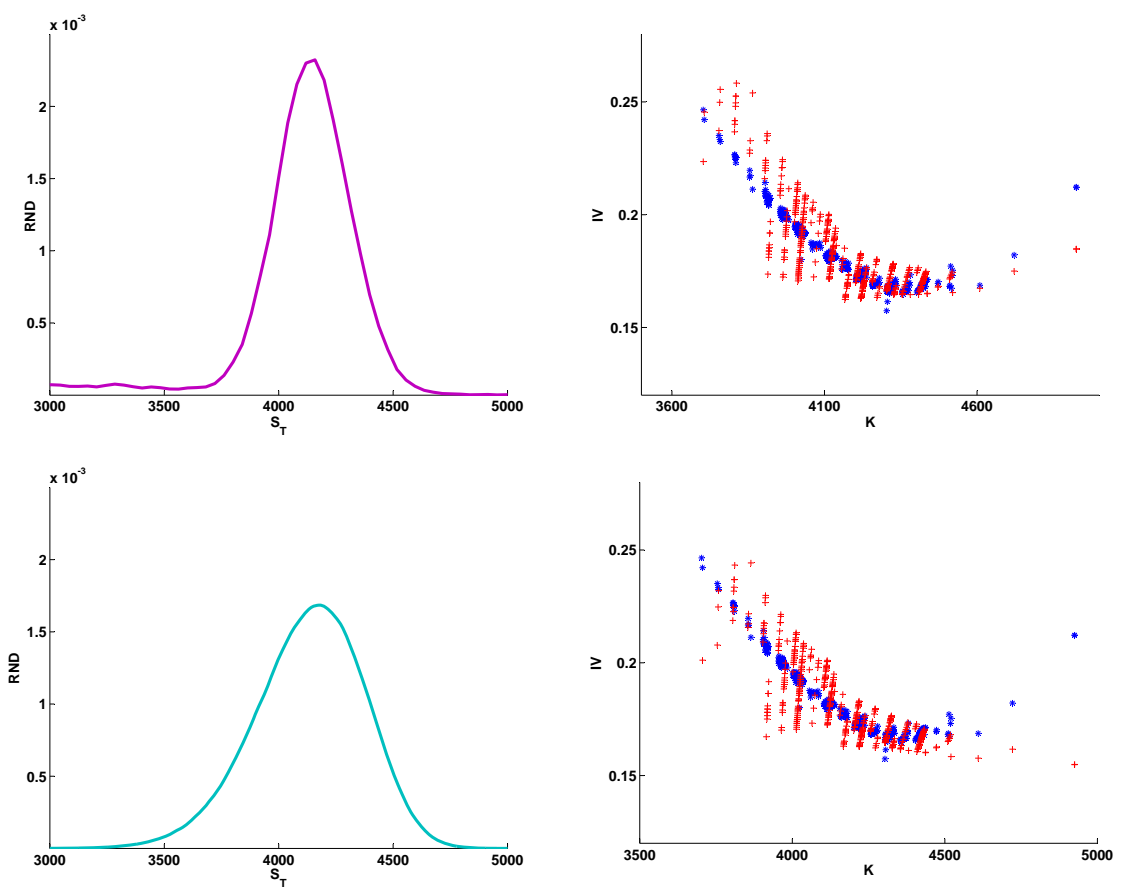

Fig. 4. Left: RND estimated for the Merton with $\sigma=0.13, \lambda=0.10, \mu=-0.23$, $\delta=0.17$ and Heston with $\theta=0.19, \rho=-0.61, \kappa=1.18, \eta=0.21$. Right: $I V_{B S}$ for observed call prices (asterisk) and fitted call prices (plus sign)

Merton model for pricing European options tries to capture the deviations from normality of log-returns by adding a compound Poisson jump process to the Black-Scholes model. Jump components add mass to the tails of the returns distribution. Increasing $\delta$ adds mass to both tails. The sign of $\mu$ determines the sign of the skewness: negative $\mu$ implies relatively more mass in the left (negative skew) and the other way around. Larger values of the intensity parameters $\lambda$ (which means that the jumps are expected to occur more frequently) makes the density flatter tailed, i.e. increases kurtosis.

In the Merton model an implied volatility skew is attainable by the presence of jumps. By introducing a correlation parameter $\rho$ between log-returns and volatility movements in the Heston model has a similar effect on the volatility smile. Varying the parameter $\rho$ around 0 gives us asymmetric tails of RND. Intuitively, if $\rho>0$, then volatility will increase as the asset price/return increases. This will spread the right tail and squeeze the left tail of the distri- 

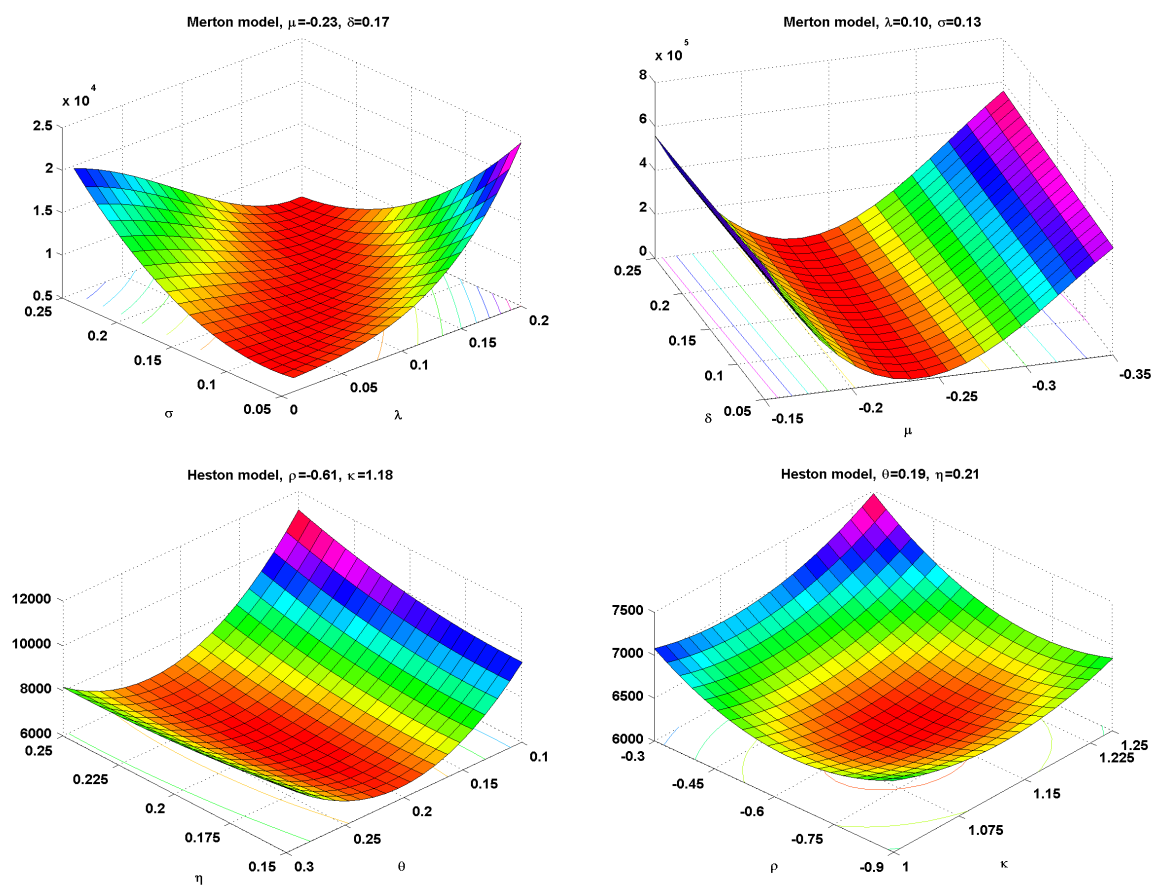

Fig. 5. Calibration error function by indirect methods

bution creating a fat right-tailed distribution. Parameter $\kappa$ measures the speed of mean reversion and can be interpreted as the degree of 'volatility clustering' in the sense that large price variations are likely to be followed by large price variations and the other way around. $\eta$ is the long run level of volatility and $\theta$ is the volatility of volatility. $\theta$ affects the kurtosis of the distribution: when it is 0 the $\log$-returns will be normally distributed. Increasing $\theta$ will then increase the kurtosis only, creating heavy tails on both sides. Conversely, if $\theta<0$, then volatility will increase when the asset price/return decreases, thus spreading the left tail and squeezing the right tail of the distribution and creating a fat left-tailed distribution.

Empirical results for the RND by both method indicate negative skewness: $\mu>0$ in Merton model and $\rho<0$ in Heston model. Negative correlation $\rho$ is in line with the empirical studies of the financial returns which show that volatility is negatively correlated with the returns. Reproducing some of the essential features of asset dynamics can result in significant shape differences. We can see in figure 4 that RND implied by Merton has a much fatter left tail and a higher kurtosis than the RND obtained from the Heston model. This shows how different models for the stock prices give various shapes of the risk neutral density. In terms of the implied volatility, Merton model seems more suitable to reproduce the skew in figure 4. Pricing errors have a very similar 


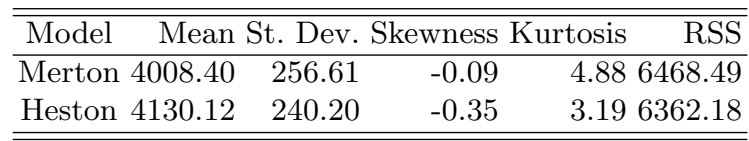

Table 2. Comparison of the RND estimates by indirect method in terms of moments and fit: Merton with $\sigma=0.13, \lambda=0.10, \mu=-0.23, \delta=0.17$ and Heston with $\theta=0.19, \rho=-0.61, \kappa=1.18, \eta=0.21$

structure for the two models: they are almost symmetrical against the 0 line and decrease for high strike prices.

The graphs in figure 5 show the calibration error function in both models for pairs of parameters in each model. Three of the panels indicate that the calibration is ill-posed because there is a large, nearly flat region or a valley of minima for the objective function. It implies that there are many parameter sets for which the model prices match the observed prices. However, by using this approach the shape of RND for different set of parameters that give a comparable good fit may differ a lot. We do not report such graphs here, but one can easily vary two of the parameters along a valley in figure 5 to verify this. The right panel bottom indicate that the objective function has a clearly defined minimum so that the pairs $(\rho, \kappa)$ in the Heston model are uniquely defined when keeping the other model parameters fixed.

In modelling the risk neutral densities based on option data the practitioners face a trade off between modelling aspects of the underlying's dynamics and reliability of calculations concerning the shape of the RND. While some distribution families allow for great flexibility in the shape of RND (e.g. generalized gamma) they are not very informative about the dynamic of the underlying asset. If modelling the underlying process is preferred indirect methods are to be chosen. The challenge is to find a model that is able the reproduce the main features of the stock prices. 


\section{References}

1. L. Andersen and J. Andreasen. Jump-diffusion models: Volatility smile fitting and numerical methods for pricing. Rev. Derivatives Research, 4:231-262, 2000.

2. B. Bahra. Implied risk-neutral probability density functions from option prices. Working paper, Bank of England, 1997.

3. David S Bates. Jumps and stochastic volatility: Exchange rate processes implicit in deutsche mark options. Review of Financial Studies, 9(1):69-107, 1996.

4. S. Biagini and R. Cont. Model-free representation of pricing rules as conditional expectations. In J. Akahori, S. Ogawa, and S. Watanabe, editors, Proceedings of the 6th Ritsumeikan International Symposium - Stochastic Processes and Applications to Mathematical Finance, pages 53-66. World Scientific,, 2006.

5. F. Black and M. Scholes. The pricing of options and corporate liabilities. Journal of Political Economy, 81:637-659, 1973.

6. P. Carr and D. Madan. Option valuation using the fast fourier transform. Journal of Computational Finance, 2:61-73, 1999.

7. P. Cizek, W. Härdle, and R. Weron. Statistical Tools in Finance and Insurance. Springer, Berlin, 2005.

8. R. Cont and P. Tankov. Financial Modelling with Jump Processes. Chapman \& Hall, 2004.

9. C.J. Corrado and T. Su. Implied volatility skew and stock index skewness and kurtosis implied by s\& p 500 index option prices. Journal of Derivatives, 4:8-19, 1997.

10. F. Delbaen and M. Schachermayer. Mathematics of Arbitrage. Springer, Berlin, 2006.

11. R. M. Dudley. Real Analysis and Probability. Cambridge University Press, Cambridge, 2002.

12. F. J. Fabozzi, R. Tunaru, and G. Albotas. Estimating risk-neutral density with parametric models in interest rate markets. Quantitative Finance, 9:55-70, 2009.

13. M. Fengler. Semiparametric Modeling of Implied Volatility. Springer, Berlin, 2005.

14. H. Föllmer and A. Schied. Stochastic Finance. de Gruyter, Berlin, 2nd edition, 2004.

15. S. Heston. A closed-form solution for options with stochastic volatility with applications to bond and currency options. Review of Financial Studies, 6(2):327343, 1993.

16. J. Hugonnier, D. Kramkov, and W. Schachermayer. On utility-based pricing of contingent claims in incomplete markets. Mathematical Finance, 15:203-212, 2005.

17. J. Jackwerth. Recovering risk aversion from option prices and realized returns. Review of Financial Studies, 13(2):433-451, 2000.

18. E. Jondeau and M. Rockinger. Reading the smile: the message conveyed by methods which infer risk neutral densities. Journal of International Money and Finance, 19:885-915, 2000.

19. R. Lee. Option pricing by transform methods: extensions, unifications and error control. Journal of Computational Finance, 7:51-86, 2004.

20. W. Melick and C. Thomas. Recovering an asset's implied pdf from option prices: An application to crude oil crisis. Journal of Financial and Quantitative Analysis, 32:91-115, 1997. 
21. R. Merton. Option pricing when underlying stock returns are discontinuous. Journal of Financial Economics, 3:125-183, 1976.

22. R. J. Ritchey. Call option valuation for discrete normal mixtures. Journal of Financial Research, 13:285-295, 1990.

23. W. Rudin. Real and Complex Analysis. McGraw-Hill, New York, 2nd ed. edition, 1974.

24. R. Savickas. A simple option-pricing formula. The Financial Review, 37:207226, 2002.

25. R. Savickas. Evidence on delta hedging and implied volatilities for black-scholes, gamma and weibull option-pricing models. Journal of Financial Research, 28:299-317, 2005.

26. P. Söderlind and L. Swensson. New techniques to extract market expectation from financial instruments. Journal of Monetary Economics, 40:383-429, 1997.

27. J. S. Walker. Fast Fourier Transforms. CRC Press, Boca Raton, 1996. 


\section{SFB 649 Discussion Paper Series 2010}

For a complete list of Discussion Papers published by the SFB 649, please visit http://sfb649. wiwi.hu-berlin. de.

001 "Volatility Investing with Variance Swaps" by Wolfgang Karl Härdle and Elena Silyakova, January 2010.

002 "Partial Linear Quantile Regression and Bootstrap Confidence Bands" by Wolfgang Karl Härdle, Ya'acov Ritov and Song Song, J anuary 2010.

003 "Uniform confidence bands for pricing kernels" by Wolfgang Karl Härdle, Yarema Okhrin and Weining Wang, January 2010.

004 "Bayesian Inference in a Stochastic Volatility Nelson-Siegel Model" by Nikolaus Hautsch and Fuyu Yang, January 2010.

005 "The I mpact of Macroeconomic News on Quote Adjustments, Noise, and Informational Volatility" by Nikolaus Hautsch, Dieter Hess and David Veredas, January 2010.

006 "Bayesian Estimation and Model Selection in the Generalised Stochastic Unit Root Model" by Fuyu Yang and Roberto Leon-Gonzalez, January 2010.

007 "Two-sided Certification: The market for Rating Agencies" by Erik R. Fasten and Dirk Hofmann, January 2010.

008 "Characterising Equilibrium Selection in Global Games with Strategic Complementarities" by Christian Basteck, Tijmen R. Daniels and Frank Heinemann, January 2010.

009 "Predicting extreme VaR: Nonparametric quantile regression with refinements from extreme value theory" by Julia Schaumburg, February 2010.

010 "On Securitization, Market Completion and Equilibrium Risk Transfer" by Ulrich Horst, Traian A. Pirvu and Gonçalo Dos Reis, February 2010.

011 "Illiquidity and Derivative Valuation" by Ulrich Horst and Felix Naujokat, February 2010.

012 "Dynamic Systems of Social Interactions" by Ulrich Horst, February 2010.

013 "The dynamics of hourly electricity prices" by Wolfgang Karl Härdle and Stefan Trück, February 2010.

014 "Crisis? What Crisis? Currency vs. Banking in the Financial Crisis of 1931" by Albrecht Ritschl and Samad Sarferaz, February 2010.

015 "Estimation of the characteristics of a Lévy process observed at arbitrary frequency" by Johanna Kappusl and Markus Reiß, February 2010.

016 "Honey, I'll Be Working Late Tonight. The Effect of Individual Work Routines on Leisure Time Synchronization of Couples" by Juliane Scheffel, February 2010.

017 "The Impact of ICT Investments on the Relative Demand for HighMedium-, and Low-Skilled Workers: Industry versus Country Analysis" by Dorothee Schneider, February 2010.

018 "Time varying Hierarchical Archimedean Copulae" by Wolfgang Karl Härdle, Ostap Okhrin and Yarema Okhrin, February 2010.

019 "Monetary Transmission Right from the Start: The (Dis)Connection Between the Money Market and the ECB's Main Refinancing Rates" by Puriya Abbassi and Dieter Nautz, March 2010.

020 "Aggregate Hazard Function in Price-Setting: A Bayesian Analysis Using Macro Data" by Fang Yao, March 2010.

021 "Nonparametric Estimation of Risk-Neutral Densities" by Maria Grith, Wolfgang Karl Härdle and Melanie Schienle, March 2010. 


\section{SFB 649 Discussion Paper Series 2010}

For a complete list of Discussion Papers published by the SFB 649, please visit http://sfb649. wiwi. hu-berlin.de.

022 "Fitting high-dimensional Copulae to Data" by Ostap Okhrin, April 2010.

023 "The (In)stability of Money Demand in the Euro Area: Lessons from a Cross-Country Analysis" by Dieter Nautz and Ulrike Rondorf, April 2010.

024 "The optimal industry structure in a vertically related market" by Raffaele Fiocco, April 2010.

025 "Herding of Institutional Traders" by Stephanie Kremer, April 2010.

026 "Non-Gaussian Component Analysis: New Ideas, New Proofs, New Applications" by Vladimir Panov, May 2010.

027 "Liquidity and Capital Requirements and the Probability of Bank Failure" by Philipp Johann König, May 2010.

028 "Social Relationships and Trust" by Christine Binzel and Dietmar Fehr, May 2010.

029 "Adaptive Interest Rate Modelling" by Mengmeng Guo and Wolfgang Karl Härdle, May 2010.

030 "Can the New Keynesian Phillips Curve Explain Inflation Gap Persistence?" by Fang Yao, June 2010.

031 "Modeling Asset Prices" by James E. Gentle and Wolfgang Karl Härdle, June 2010.

032 "Learning Machines Supporting Bankruptcy Prediction" by Wolfgang Karl Härdle, Rouslan Moro and Linda Hoffmann, June 2010.

033 "Sensitivity of risk measures with respect to the normal approximation of total claim distributions" by Volker Krätschmer and Henryk Zähle, June 2010.

034 "Sociodemographic, Economic, and Psychological Drivers of the Demand for Life Insurance: Evidence from the German Retirement Income Act" by Carolin Hecht and Katja Hanewald, July 2010.

035 "Efficiency and Equilibria in Games of Optimal Derivative Design" by Ulrich Horst and Santiago Moreno-Bromberg, July 2010.

036 "Why Do Financial Market Experts Misperceive Future Monetary Policy Decisions?" by Sandra Schmidt and Dieter Nautz, July 2010.

037 "Dynamical systems forced by shot noise as a new paradigm in the interest rate modeling" by Alexander L. Baranovski, July 2010.

038 "Pre-Averaging Based Estimation of Quadratic Variation in the Presence of Noise and Jumps: Theory, Implementation, and Empirical Evidence" by Nikolaus Hautsch and Mark Podolskij, July 2010.

039 "High Dimensional Nonstationary Time Series Modelling with Generalized Dynamic Semiparametric Factor Model" by Song Song, Wolfgang K. Härdle, and Ya'acov Ritov, July 2010.

040 "Stochastic Mortality, Subjective Survival Expectations, and Individual Saving Behavior" by Thomas Post and Katja Hanewald, J uly 2010.

041 "Prognose mit nichtparametrischen Verfahren" by Wolfgang Karl Härdle, Rainer Schulz, and Weining Wang, August 2010.

042 "Payroll Taxes, Social Insurance and Business Cycles" by Michael C. Burda and Mark Weder, August 2010.

043 "Meteorological forecasts and the pricing of weather derivatives" by Matthias Ritter, Oliver Mußhoff, and Martin Odening, September 2010.

044 "The High Sensitivity of Employment to Agency Costs: The Relevance of Wage Rigidity" by Atanas Hristov, September 2010.

045 "Parametric estimation of risk neutral density functions" by Maria Grith and Volker Krätschmer, September 2010. 